\title{
XCS Classifier System with Experience Replay
}

\author{
Supplemental Material
}

ANTHONY STEIN, University of Hohenheim, Germany

ROLAND MAIER, University of Augsburg, Germany

LUKAS ROSENBAUER, BSH Home Appliances, Germany

JÖRG HÄHNER, University of Augsburg, Germany

\section{A EXPERIMENT CONFIGURATIONS}

Environment Modifications. For the n-Chain environment, the episode length was reduced from 1000 to only 200 , what increases the problem difficulty.

For the CartPole environment, the input parameters have been re-scaled to the interval [0,1] (using reasonable lower and upper bounds as determined in preliminary runs) since the utilized XCS implementation expects normalized inputs. The reward scheme was slightly modified to deliver +1 every time the pole keeps balanced by the cart and 0 if it has fallen. This provides a clearer reward signal and facilitates quicker learning for each of the compared algorithms.

Regarding the MountainCar environment, we again changed the episode length from 200 to 500, since both XCS variants encountered problems in randomly stumbling into the goal state within only 200 steps. Further, the reward scheme was made binary, i.e., instead of paying -1 for every step (includes reaching the terminal/goal state), we chose to apply a $0 / 1000$ scheme. The latter only pays a reward of 1000 when reaching the goal, otherwise 0 . This modification essentially increases the problem difficulty, since it renders the reward signal sparse.

Authors' addresses: Anthony Stein, University of Hohenheim, Germany, anthony.stein@uni-hohenheim.de; Roland Maier, University of Augsburg, Germany, roland.maximilian.maier@student.uni-augsburg.de; Lukas Rosenbauer, BSH Home Appliances, Germany, lukas.rosenbauer@bshg.com; Jörg Hähner, University of Augsburg, Germany, joerg.haehner@informatik.uni-augsburg.de. 
Table 1. Configured hyperparameter settings. Abbreviations used: TS $=$ tournament selection, UBR $=$ unordered bound hyperrectangular representation, $\mathrm{WH}=$ Widrow-Hoff learning rule, $\mathrm{RLS}=$ recursive least square learning rule

\begin{tabular}{|c|c|c|c|c|c|c|}
\hline Parameter & Mario & 6-RMP & WBC & 16chain & CartPole & M.Car \\
\hline Learning steps & $100 \mathrm{k}$ & $40 \mathrm{k}$ & $50 \mathrm{k}$ & $50 \mathrm{k}$ & $50 \mathrm{k}$ & $60 \mathrm{k}$ \\
\hline$N_{\max }$ & 7000 & 800 & 6400 & 1000 & 5000 & 3000 \\
\hline$\beta$ & 0.3 & 0.2 & 0.2 & 0.1 & 0.1 & 0.1 \\
\hline$\gamma$ & - & - & - & 0.9 & 0.99 & 0.99 \\
\hline \multicolumn{7}{|l|}{ Fitness parameters: } \\
\hline$\alpha$ & 0.1 & 0.1 & 0.1 & 0.1 & 0.1 & 0.1 \\
\hline$\epsilon_{0}$ & 10 & 10 & 1 & 0.1 & 8.0 & 0.1 \\
\hline$v$ & 5 & 5 & 5 & 5 & 5 & 5 \\
\hline \multicolumn{7}{|l|}{ Deletion parameters: } \\
\hline$\theta_{d e l}$ & 50 & 20 & 50 & 20 & 200 & 200 \\
\hline$\delta$ & 0.1 & 0.1 & 0.1 & 0.1 & 0.1 & 0.1 \\
\hline \multicolumn{7}{|l|}{ Covering parameters: } \\
\hline$\theta_{m n a}$ & 7 & 2 & 2 & 2 & 2 & 3 \\
\hline$p_{i n i}$ & 10 & 10 & 10 & 10 & 0.01 & 0.01 \\
\hline$\epsilon_{i n i}$ & 0 & 0 & 0 & 10 & 0.01 & 0.01 \\
\hline$F_{\text {ini }}$ & 0.01 & 0.01 & 0.01 & 0.01 & 0.01 & 0.01 \\
\hline \multicolumn{7}{|l|}{ GA parameters: } \\
\hline$\mu$ & 0.04 & 0.04 & 0.04 & 0.04 & 0.04 & 0.04 \\
\hline$\chi$ & 0.8 & 0.8 & 0.8 & 0.8 & 0.8 & 0.8 \\
\hline$\theta_{G A}$ & 30 & 12 & 48 & 50 & 25 & 50 \\
\hline$\theta_{\text {sub }}$ & 50 & 20 & 50 & 200 & 20 & 20 \\
\hline GA-selection & TS & TS & TS & TS & TS & TS \\
\hline$F_{\text {reduce }}$ & 0.1 & 0.1 & 0.1 & 0.1 & 0.1 & 0.1 \\
\hline$\epsilon_{\text {reduce }}$ & 1.0 & 1.0 & 1.0 & 0.25 & 0.25 & 0.25 \\
\hline \multicolumn{7}{|l|}{ Condition parameters: } \\
\hline Condition & UBR & UBR & UBR & UBR & UBR & UBR \\
\hline$m_{0}$ & 0.1 & 0.1 & 0.2 & 0.1 & 0.1 & 0.1 \\
\hline$r_{0}$ & 0.1 & 1.0 & 0.4 & 0.2 & 0.2 & 0.33 \\
\hline \multicolumn{7}{|l|}{ Prediction parameters: } \\
\hline Prediction & scalar & scalar & scalar & scalar & linear & linear \\
\hline Learning rule & WH & WH & WH & WH & RLS & RLS \\
\hline$\delta_{R L S}$ & - & - & - & - & 1.0 & 500 \\
\hline$\lambda_{R L S}$ & - & - & - & - & 1.0 & 1.0 \\
\hline
\end{tabular}

For all experiments a maximum capacity for the Replay Memory (RM) of $N=50 k$ has been chosen. The mini-batch size was set to $m=4$. In all experiments, a warm-up phase to fill the RM was conducted during the initial 1000 steps. No learning happened in this phase. 


\section{B ADDITIONAL STATISTICS}

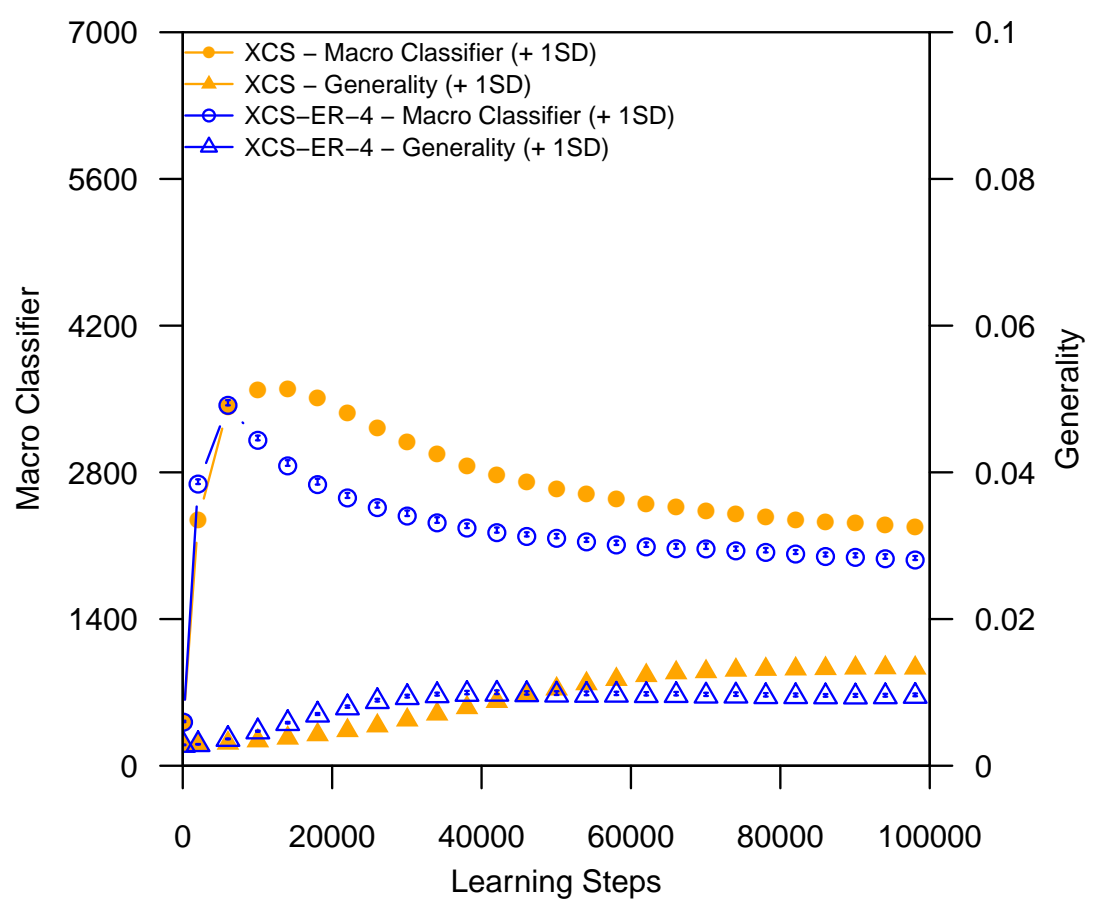

Fig. 1. XCS vs. XCS-ER on Mario pixel art.

Table 2. Shapiro-Wilk tests for normality on the Mario problem. The resulting p-values are given in the cells. ${ }^{*}\left({ }^{* *}\right)$ indicates a p-value $<\alpha=0.05$ (0.01), i.e. a high likelihood that the null hypothesis (normality) can be rejected. p-values below 0.05 are marked in bold.

\begin{tabular}{|l|l|l|l|}
\cline { 2 - 4 } \multicolumn{1}{l|}{ Mario } & XCS-ER-8 & XCS-ER-4 & XCS \\
\hline Reward mean & 0.783 & 0.637 & 0.936 \\
\hline System error mean & 0.467 & 0.858 & 0.065 \\
\hline Macroclassifiers mean & 0.209 & 0.863 & 0.235 \\
\hline Generality mean & 0.213 & 0.586 & 0.110 \\
\hline
\end{tabular}

Table 3. Paired one-sided t-tests for XCS vs. XCS-ER on the Mario problem. The resulting p-values are given in the cells. ${ }^{*}\left({ }^{* *}\right)$ indicates a p-value $<\alpha=0.05$ (0.01), i.e. a high likelihood the deviation is statistically significant. p-values below 0.05 are marked in bold.

\begin{tabular}{|l|l|l|}
\cline { 2 - 3 } \multicolumn{1}{l|}{ Mario } & XCS-ER-8 & XCS-ER-4 \\
\hline Reward mean & $\mathbf{0 . 0 0 0}^{* *}$ & $\mathbf{0 . 0 0 0}^{* *}$ \\
\hline System error mean & $\mathbf{0 . 0 0 0}^{* *}$ & $\mathbf{0 . 0 0 0}^{* *}$ \\
\hline Macroclassifiers mean & $\mathbf{0 . 0 0 0}^{* *}$ & $\mathbf{0 . 0 0 0}^{* *}$ \\
\hline Generality mean & $\mathbf{0 . 0 0 0}^{* *}$ & $\mathbf{0 . 0 0 0}^{* *}$ \\
\hline
\end{tabular}




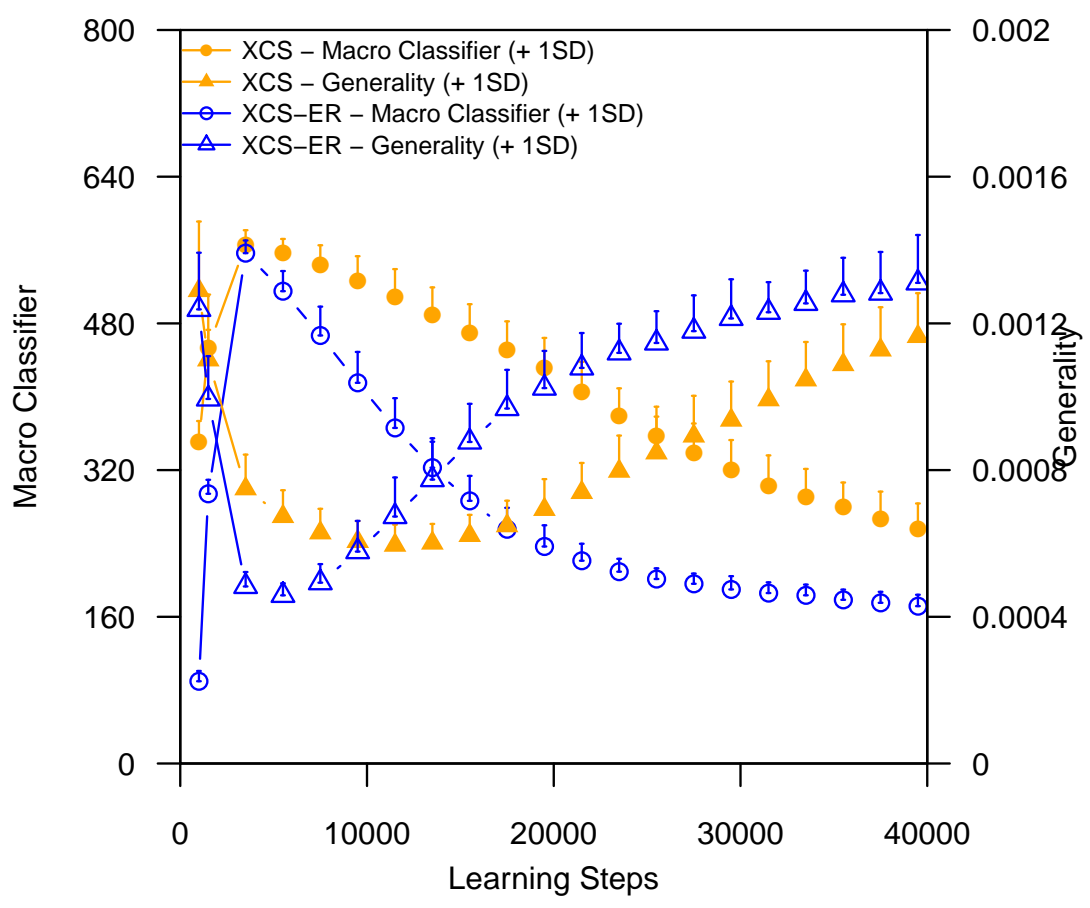

Fig. 2. XCS vs. XCS-ER on 6-Real Multiplexer

Table 4. Shapiro-Wilk tests for normality for the 6-RMP problem. The resulting p-values are given in the cells. * $\left({ }^{* *}\right)$ indicates a $\mathrm{p}$-value $<\alpha=0.05(0.01)$, i.e. a high likelihood that the null hypothesis (normality) can be rejected. $\mathrm{p}$-values below 0.05 are marked in bold.

\begin{tabular}{|l|l|l|}
\cline { 2 - 3 } \multicolumn{1}{l|}{ 6-Real Multiplexer } & XCS-ER & XCS \\
\hline Reward mean & 0.060 & 0.872 \\
\hline System error mean & 0.143 & 0.714 \\
\hline Macroclassifiers mean & $\mathbf{0 . 0 3 7}^{*}$ & 0.882 \\
\hline Generality mean & 0.846 & 0.117 \\
\hline
\end{tabular}

Table 5. Paired one-sided t-tests for XCS vs. XCS-ER on the 6-RMP problem. The resulting p-values are given in the cells. ${ }^{*}\left({ }^{* *}\right)$ indicates a p-value $<\alpha=0.05$ (0.01), i.e. a high likelihood the deviation from the baseline (XCS without ER) is statistically significant. p-values below 0.05 are marked in bold.

\begin{tabular}{|l|l|}
\cline { 2 - 2 } \multicolumn{1}{l|}{ 6-Real Multiplexer } & XCS-ER \\
\hline Reward mean & $\mathbf{0 . 0 0 0}^{* *}$ \\
\hline System error mean & $\mathbf{0 . 0 0 0}^{* *}$ \\
\hline Macroclassifiers mean & $\mathbf{0 . 0 0 0}^{* *}$ \\
\hline Generality mean & $\mathbf{0 . 0 0 0}^{* *}$ \\
\hline
\end{tabular}




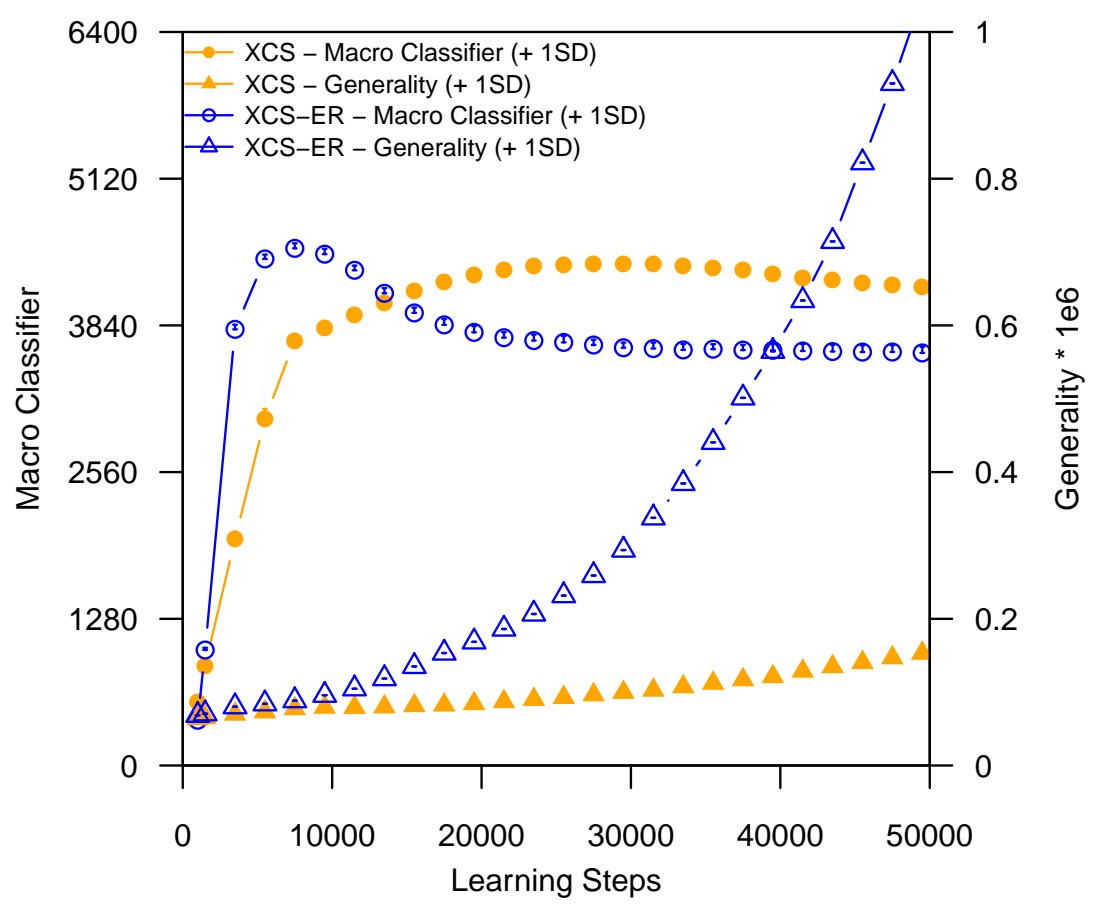

Fig. 3. XCS vs. XCS-ER on WBC classification

Table 6. Shapiro-Wilk tests for normality on the WBC data set. The resulting p-values are depicted in the cells. ${ }^{*}\left({ }^{* *}\right)$ indicates a $\mathrm{p}$-value $<\alpha=0.05$ (0.01), i.e. a high likelihood that the null hypothesis (normality) can be rejected. p-values below 0.05 are marked in bold.

\begin{tabular}{|l|l|l|}
\cline { 2 - 3 } \multicolumn{1}{l|}{ WBC } & XCS-ER & XCS \\
\hline Reward mean & 0.458 & 0.333 \\
\hline System error mean & 0.195 & 0.316 \\
\hline Macroclassifiers mean & 0.096 & 0.458 \\
\hline Generality mean & $\mathbf{0 . 0 2 6}^{\text {* }}$ & $\mathbf{0 . 0 0 3}^{\text {** }}$ \\
\hline
\end{tabular}

Table 7. Paired one-sided t-tests for XCS vs. XCS-ER on the WBC data set. The resulting p-values are given in the cells. $\left.{ }^{* * *}\right)$ indicates a p-value $<\alpha=0.05$ (0.01), i.e. a high likelihood the deviation from the baseline (XCS without ER) is statistically significant. p-values below 0.05 are marked in bold.

\begin{tabular}{|l|l|}
\cline { 2 - 2 } \multicolumn{1}{l|}{ WBC } & XCS-ER \\
\hline Reward mean & $\mathbf{0 . 0 0 0}^{* *}$ \\
\hline System error mean & $\mathbf{0 . 0 0 0}^{* *}$ \\
\hline Macroclassifiers mean & $\mathbf{0 . 0 0 0}^{* *}$ \\
\hline Generality mean & $\mathbf{0 . 0 0 0}^{* *}$ \\
\hline
\end{tabular}


Table 8. Overall results for the multi-step environments: Reward, prediction error, population size in macroclassifiers, generality, OTM and Divergences are shown. Table entries indicate the overall means $\pm 1 S D$ over the 30 repetitions. Divergence criteria for individual repetitions for 16Chain, CartPole, MountainCar are OTM values below 1, 70 and 200 respectively. 'T' indicates active teletransportation. Arrows show whether the metric increased up or decreased. Statistical significance when compared to the regular XCS is determined via the Wilcoxon signed-rank test. ${ }^{*}\left({ }^{* *}\right)$ indicates a p-value $<\alpha=0.05(0.01)$. p-values below 0.05 confirming improvements are given in bold.

\begin{tabular}{|c|c|c|c|c|c|c|}
\hline 16Chain & Reward & Pr.Err. & $|[P]|$ & Gen. & OTM & Div \\
\hline XCS-ER & $\begin{array}{l}0.56 \downarrow^{* *} \\
\pm 0.37\end{array}$ & $\begin{array}{l}0.23 \uparrow^{* *} \\
\pm 0.01\end{array}$ & $\begin{array}{l}92.92 \downarrow^{* *} \\
\pm 25.81\end{array}$ & $\begin{array}{l}0.13 \uparrow^{* *} \\
\pm 0.06\end{array}$ & $\begin{array}{l}95.3 \downarrow^{* *} \\
\pm 63.6\end{array}$ & $9 \uparrow$ \\
\hline DQN & $\mathrm{n} / \mathrm{a}$ & $\mathrm{n} / \mathrm{a}$ & $\mathrm{n} / \mathrm{a}$ & $\mathrm{n} / \mathrm{a}$ & $\begin{array}{l}143.1 \uparrow \\
\pm 1.4\end{array}$ & $0 \downarrow$ \\
\hline $\mathrm{XCS}$ & $\begin{array}{l}0.82 \\
\pm 0.15\end{array}$ & $\begin{array}{l}0.14 \\
\pm 0.03\end{array}$ & $\begin{array}{l}152.68 \\
\pm 22.16\end{array}$ & $\begin{array}{l}0.07 \\
\pm 0.02\end{array}$ & $\begin{array}{l}138.6 \\
\pm 25.7\end{array}$ & 1 \\
\hline 16Chain T & Reward & Pr.Err. & $|[P]|$ & Gen. & OTM & $\overline{\text { Div }}$ \\
\hline XCST-ER & $\begin{array}{l}0.86 \downarrow \\
\pm 0.03\end{array}$ & $\begin{array}{l}0.24 \uparrow^{* *} \\
\pm 0.01\end{array}$ & $\begin{array}{l}111.65 \downarrow \\
\pm 21.94\end{array}$ & $\begin{array}{l}0.10 \uparrow \\
\pm 0.05\end{array}$ & $\begin{array}{l}147.6 \downarrow \\
\pm 4.2\end{array}$ & 0 \\
\hline DQNT & $\mathrm{n} / \mathrm{a}$ & $\mathrm{n} / \mathrm{a}$ & $\mathrm{n} / \mathrm{a}$ & $\mathrm{n} / \mathrm{a}$ & $\begin{array}{l}148.0 \downarrow \\
\pm 5.8 \\
\end{array}$ & 0 \\
\hline $\mathrm{XCST}$ & $\begin{array}{l}0.88 \\
\pm 0.00\end{array}$ & $\begin{array}{l}0.20 \\
\pm 0.03\end{array}$ & $\begin{array}{l}117.90 \\
\pm 13.54\end{array}$ & $\begin{array}{l}0.09 \\
\pm 0.01 \\
\end{array}$ & $\begin{array}{l}149.2 \\
\pm 0.6 \\
\end{array}$ & 0 \\
\hline CartPole & Reward & Pr.Err. & $|[P]|$ & Gen. & OTM & Div \\
\hline XCS-ER & $\begin{array}{l}0.98 \downarrow \\
\pm 0.03\end{array}$ & $\begin{array}{l}1.92 \uparrow^{* *} \\
\pm 0.11\end{array}$ & $\begin{array}{l}1937.44 \uparrow^{* *} \\
\pm 106.85\end{array}$ & $\begin{array}{l}0.0004 \uparrow^{* *} \\
\pm 0.0000\end{array}$ & $\begin{array}{l}93.0 \downarrow \\
\pm 47.6\end{array}$ & $9 \uparrow$ \\
\hline DQN & $\mathrm{n} / \mathrm{a}$ & $\mathrm{n} / \mathrm{a}$ & $\mathrm{n} / \mathrm{a}$ & $\mathrm{n} / \mathrm{a}$ & $\begin{array}{l}122.2 \uparrow \\
\pm 13.4 \\
\end{array}$ & $0 \downarrow$ \\
\hline $\mathrm{XCS}$ & $\begin{array}{l}0.99 \\
\pm 0.01\end{array}$ & $\begin{array}{l}1.62 \\
\pm 0.25\end{array}$ & $\begin{array}{l}1703.42 \\
\pm 161.46\end{array}$ & $\begin{array}{l}0.0003 \\
\pm 0.0000\end{array}$ & $\begin{array}{l}110.6 \\
\pm 46.5\end{array}$ & 7 \\
\hline CartPole T & Reward & Pr.Err. & $|[P]|$ & Gen. & OTM & Div \\
\hline XCST-ER & $\begin{array}{l}0.94 \uparrow^{* *} \\
\pm 0.02\end{array}$ & $\begin{array}{l}2.17 \uparrow^{* *} \\
\pm 0.12\end{array}$ & $\begin{array}{l}2162.30 \downarrow^{* *} \\
\pm 51.94\end{array}$ & $\begin{array}{l}0.0013 \uparrow^{* *} \\
\pm 0.0000\end{array}$ & $\begin{array}{l}18.29 \uparrow^{* *} \\
\pm 6.22\end{array}$ & 30 \\
\hline DQNT & $\mathrm{n} / \mathrm{a}$ & $\mathrm{n} / \mathrm{a}$ & $\mathrm{n} / \mathrm{a}$ & $\mathrm{n} / \mathrm{a}$ & $\begin{array}{l}38.75^{* *} \\
\pm 2.19\end{array}$ & 30 \\
\hline $\mathrm{XCST}$ & $\begin{array}{l}0.92 \\
\pm 0.02\end{array}$ & $\begin{array}{l}1.90 \\
\pm 0.09\end{array}$ & $\begin{array}{l}2640.10 \\
\pm 70.37\end{array}$ & $\begin{array}{l}0.0005 \\
\pm 0.0001\end{array}$ & $\begin{array}{l}13.76 \\
\pm 3.59\end{array}$ & 30 \\
\hline M.Car & Reward & Pr.Err. & $|[P]|$ & Gen. & OTM & Div \\
\hline XCS-ER & $\begin{array}{l}0.13 \uparrow \\
\pm 0.06\end{array}$ & $\begin{array}{l}1.73 \downarrow^{* *} \\
\pm 2.77\end{array}$ & $\begin{array}{l}383.25 \uparrow \\
\pm 204.68\end{array}$ & $\begin{array}{l}0.05 \uparrow^{* *} \\
\pm 0.01\end{array}$ & $\begin{array}{l}38.57 \uparrow \\
\pm 86.37\end{array}$ & 29 \\
\hline $\mathrm{DQN}$ & $\mathrm{n} / \mathrm{a}$ & $\mathrm{n} / \mathrm{a}$ & $\mathrm{n} / \mathrm{a}$ & $\mathrm{n} / \mathrm{a}$ & $\begin{array}{l}504.96 \uparrow^{* *} \\
\pm 216.03\end{array}$ & $3 \downarrow$ \\
\hline $\mathrm{XCS}$ & $\begin{array}{l}0.06 \\
\pm 0.15\end{array}$ & $\begin{array}{l}3.5 \mathrm{E} 5 \\
\pm 1.8 \mathrm{E} 6\end{array}$ & $\begin{array}{l}325.17 \\
\pm 162.39\end{array}$ & $\begin{array}{l}0.04 \\
\pm 0.01\end{array}$ & $\begin{array}{l}29.15 \\
\pm 63.27\end{array}$ & 29 \\
\hline M.Car T & Reward & Pr.Err. & $|[P]|$ & Gen. & OTM & Div \\
\hline XCST-ER & $\begin{array}{l}4.33 \uparrow^{*} \\
\pm 1.3\end{array}$ & $\begin{array}{l}12.40 \downarrow^{* *} \\
\pm 3.69\end{array}$ & $\begin{array}{l}1150.36 \uparrow \\
\pm 88.24 \\
\end{array}$ & $\begin{array}{l}0.01 \downarrow^{* *} \\
\pm 0.00\end{array}$ & $\begin{array}{l}626.3 \uparrow \\
\pm 132.4 \\
\end{array}$ & $0 \downarrow$ \\
\hline DQNT & $\mathrm{n} / \mathrm{a}$ & $\mathrm{n} / \mathrm{a}$ & $\mathrm{n} / \mathrm{a}$ & $\mathrm{n} / \mathrm{a}$ & $\begin{array}{l}814.5 \uparrow^{* *} \\
\pm 90.9\end{array}$ & $0 \downarrow$ \\
\hline $\mathrm{XCST}$ & $\begin{array}{l}3.22 \\
\pm 2.17\end{array}$ & $\begin{array}{l}1.2 \mathrm{E} 21 \\
\pm 6.3 \mathrm{E} 21\end{array}$ & $\begin{array}{l}1049.51 \\
\pm 114.82\end{array}$ & $\begin{array}{l}0.02 \\
\pm 0.00\end{array}$ & $\begin{array}{l}511.2 \\
\pm 219.5\end{array}$ & 5 \\
\hline
\end{tabular}


Table 9. Shapiro-Wilk tests for normality on the multi-step environments. The resulting p-values are given in the cells. * $\left({ }^{* *}\right)$ indicates a p-value $<\alpha=0.05(0.01)$, i.e. a high likelihood that the null hypothesis (normality) can be rejected. p-values below 0.05 are marked in bold.

\begin{tabular}{|c|c|c|c|}
\hline 16Chain & XCS-ER & $\mathrm{XCS}$ & DQN \\
\hline Reward mean & $0.000^{* *}$ & $0.000^{* *}$ & $\mathrm{n} / \mathrm{a}$ \\
\hline Prediction error mean & $0.003^{* *}$ & 0.366 & $\mathrm{n} / \mathrm{a}$ \\
\hline Macroclassifiers mean & 0.058 & $0.008^{* *}$ & $\mathrm{n} / \mathrm{a}$ \\
\hline Generality mean & $0.000^{* *}$ & $0.000^{* *}$ & $\mathrm{n} / \mathrm{a}$ \\
\hline OTM & $0.000^{* *}$ & $0.000^{* *}$ & $0.000^{* *}$ \\
\hline 16Chain Tele. & XCS-ER & $\mathrm{XCS}$ & DQN \\
\hline Reward mean & $0.000^{* *}$ & $0.037^{*}$ & $\mathrm{n} / \mathrm{a}$ \\
\hline Prediction error mean & $\mathrm{0.001}^{* *}$ & 0.531 & $\mathrm{n} / \mathrm{a}$ \\
\hline Macroclassifiers mean & $0.000^{* *}$ & 0.597 & $\mathrm{n} / \mathrm{a}$ \\
\hline Generality mean & $0.000^{* *}$ & 0.733 & $\mathrm{n} / \mathrm{a}$ \\
\hline OTM & $0.000^{* *}$ & $0.02^{*}$ & $0.000^{* *}$ \\
\hline CartPole & XCS-ER & $\mathrm{XCS}$ & DQN \\
\hline Reward mean & $0.000^{* *}$ & $0.000^{* *}$ & $\mathrm{n} / \mathrm{a}$ \\
\hline Prediction error mean & 0.625 & $\mathbf{0 . 0 2 3}^{*}$ & $\mathrm{n} / \mathrm{a}$ \\
\hline Macroclassifiers mean & 0.875 & 0.222 & $\mathrm{n} / \mathrm{a}$ \\
\hline Generality mean & 0.432 & 0.618 & $\mathrm{n} / \mathrm{a}$ \\
\hline OTM & $0.006^{* *}$ & $0.000^{* *}$ & 0.099 \\
\hline CartPole Tele. & XCS-ER & $\mathrm{XCS}$ & DQN \\
\hline Reward mean & 0.056 & 0.639 & $\mathrm{n} / \mathrm{a}$ \\
\hline Prediction error mean & 0.184 & 0.167 & $\mathrm{n} / \mathrm{a}$ \\
\hline Macroclassifiers mean & $0.026^{*}$ & 0.976 & $\mathrm{n} / \mathrm{a}$ \\
\hline Generality mean & 0.594 & 0.240 & $\mathrm{n} / \mathrm{a}$ \\
\hline OTM & 0.177 & $0.002^{* *}$ & 0.370 \\
\hline MountainCar & XCS-ER & $\mathrm{XCS}$ & DQN \\
\hline Reward mean & $0.000^{* *}$ & $0.000^{* *}$ & $\mathrm{n} / \mathrm{a}$ \\
\hline Prediction error mean & $0.000^{* *}$ & $0.000^{* *}$ & $\mathrm{n} / \mathrm{a}$ \\
\hline Macroclassifiers mean & $0.000^{* *}$ & $0.006^{* *}$ & $\mathrm{n} / \mathrm{a}$ \\
\hline Generality mean & 0.367 & 0.303 & $\mathrm{n} / \mathrm{a}$ \\
\hline OTM & $0.000^{* *}$ & $0.000^{* *}$ & $0.023^{*}$ \\
\hline MountainCar Tele. & XCS-ER & $\mathrm{XCS}$ & DQN \\
\hline Reward mean & 0.673 & 0.067 & $\mathrm{n} / \mathrm{a}$ \\
\hline Prediction error mean & 0.641 & $0.000^{* *}$ & $\mathrm{n} / \mathrm{a}$ \\
\hline Macroclassifiers mean & $\mathrm{0.024}^{*}$ & $0^{2.023}{ }^{*}$ & $\mathrm{n} / \mathrm{a}$ \\
\hline Generality mean & 0.070 & $0.000^{* *}$ & $\mathrm{n} / \mathrm{a}$ \\
\hline OTM & $0.004^{* *}$ & 0.042 & $0.005^{* *}$ \\
\hline
\end{tabular}

As can be noticed, most of the metrics do not follow a normal distribution. Even if normality can be confirmed in certain cases, a parametric test is still not plausibly applicable when the sample considered for comparison violates this assumption. For that reason, we decided to exclusively conduct non-parametric Wilcoxon tests across all multi-step experiments. 
Table 10. Wilcoxon signed-rank tests for the multi-step environments. The resulting $p$-values are given in the cells. ${ }^{*}\left({ }^{* *}\right)$ indicates a p-value $<\alpha=0.05$ (0.01), i.e. a high likelihood that the deviation from the baseline (XCS without ER) is statistically significant. p-values below 0.05 are marked in bold.

\begin{tabular}{|c|c|c|}
\hline 16Chain & XCS-ER & DQN \\
\hline Reward mean & $0.000^{* *}$ & $\widetilde{\mathrm{n} / \mathrm{a}}$ \\
\hline Prediction error mean & $0.000^{* *}$ & $\mathrm{n} / \mathrm{a}$ \\
\hline Macroclassifiers mean & $0.000^{* *}$ & $\mathrm{n} / \mathrm{a}$ \\
\hline Generality mean & $0.000^{* *}$ & $\mathrm{n} / \mathrm{a}$ \\
\hline OTM & $0.000^{* *}$ & 0.959 \\
\hline 16Chain Tele. & XCS-ER & DQN \\
\hline Reward mean & 0.254 & $\tilde{n} / \mathrm{a}$ \\
\hline Prediction error mean & $0.000^{* *}$ & $\mathrm{n} / \mathrm{a}$ \\
\hline Macroclassifiers mean & 0.349 & $\mathrm{n} / \mathrm{a}$ \\
\hline Generality mean & 0.644 & $\mathrm{n} / \mathrm{a}$ \\
\hline OTM & 0.394 & 0.504 \\
\hline CartPole & XCS-ER & DQN \\
\hline Reward mean & 0.136 & $\mathrm{n} / \mathrm{a}$ \\
\hline Prediction error mean & $0.000^{* *}$ & $\mathrm{n} / \mathrm{a}$ \\
\hline Macroclassifiers mean & $0.000^{* *}$ & $\mathrm{n} / \mathrm{a}$ \\
\hline Generality mean & $0.000^{* *}$ & $\mathrm{n} / \mathrm{a}$ \\
\hline OTM & 0.111 & 0.428 \\
\hline CartPole Tele. & XCS-ER & DQN \\
\hline Reward mean & $0.000^{* *}$ & $\tilde{\mathrm{n} / \mathrm{a}}$ \\
\hline Prediction error mean & $0.000^{* *}$ & $\mathrm{n} / \mathrm{a}$ \\
\hline Macroclassifiers mean & $0.000^{* *}$ & $\mathrm{n} / \mathrm{a}$ \\
\hline Generality mean & $0.000^{* *}$ & $\mathrm{n} / \mathrm{a}$ \\
\hline OTM & $0.000^{* *}$ & $0.000^{* *}$ \\
\hline MountainCar & XCS-ER & DQN \\
\hline Reward mean & 0.178 & $\mathrm{n} / \mathrm{a}$ \\
\hline Prediction error mean & $0.002^{* *}$ & $\mathrm{n} / \mathrm{a}$ \\
\hline Macroclassifiers mean & 0.360 & $\mathrm{n} / \mathrm{a}$ \\
\hline Generality mean & $0.001^{* *}$ & $\mathrm{n} / \mathrm{a}$ \\
\hline OTM & 0.550 & $0.000^{* *}$ \\
\hline MountainCar Tele. & XCS-ER & DQN \\
\hline Reward mean & $\mathbf{0 . 0 1 4}^{*}$ & $\mathrm{n} / \mathrm{a}$ \\
\hline Prediction error mean & $0.000^{* *}$ & $\mathrm{n} / \mathrm{a}$ \\
\hline Macroclassifiers mean & $0.000^{* *}$ & $\mathrm{n} / \mathrm{a}$ \\
\hline Generality mean & $0.000^{* *}$ & $\mathrm{n} / \mathrm{a}$ \\
\hline OTM & 0.060 & $0.000^{* *}$ \\
\hline
\end{tabular}

